\title{
Functional Tests Vendor Name
}

National Cancer Institute

\section{Source}

National Cancer Institute. Functional Tests Vendor Name. NCI Thesaurus. Code C162135.

The literal identifier of the vendor or laboratory that performs a functional test assessment. 